\title{
Wazāra, the killer of many husbands
}

Le Caire, Institut français d'archéologie orientale (collection Textes arabes et études islamiques), 2007, 412 p. bibliographie, index, cartes. [ISBN 978-2-7247-0475-4]

\section{Katia Zakharia}

\section{OpenEdition}

\section{Journals}

Édition électronique

URL : http://journals.openedition.org/beo/219

DOI : $10.4000 /$ beo.219

ISBN : 978-2-35159-318-9

ISSN : 2077-4079

\section{Éditeur}

Presses de l'Institut français du Proche-Orient

Édition imprimée

Date de publication : 1 octobre 2010

Pagination : 165-169

ISBN : 978-2-35159-170-3

ISSN : 0253-1623

\section{Référence électronique}

Katia Zakharia, «Wazāra, the killer of many husbands », Bulletin d'études orientales [En ligne], Tome LIX | octobre 2010, mis en ligne le 01 octobre 2011, consulté le 22 septembre 2020. URL : http:// journals.openedition.org/beo/219; DOI : https://doi.org/10.4000/beo.219

Ce document a été généré automatiquement le 22 septembre 2020.

(c) Institut français du Proche-Orient 


\section{Wazāra, the killer of many husbands}

Le Caire, Institut français d'archéologie orientale (collection Textes arabes et études islamiques), 2007, 412 p. bibliographie, index, cartes. [ISBN 978-2-7247-0475-4]

\section{Katia Zakharia}

\section{RÉFÉRENCE}

Wazāra, the killer of many husbands. Le Caire, Institut français d'archéologie orientale (collection Textes arabes et études islamiques), 2007, 412 p. bibliographie, index, cartes.

[ISBN 978-2-7247-0475-4]

Pieter Smoor reprend à son compte, dans le titre de son ouvrage, Wazāra, the killer of many husbands, une métaphore récurrente dans l'œuvre poétique d'Abū Muhammad 'Umāra ibn 'Alī (m. 1174), qui personnifie la wazāra pour en faire, selon les cas, une jouvencelle voilée, une fiancée attirante ou capricieuse, une épouse stérile ou une veuve noire, mettant à profit le fait qu'en langue arabe le terme wazāra (i. e. vizirat)est de genre féminin. L'ouvrage se fonde en effet sur les panégyriques composés par ce poète, sur une période de dix ans (549/1154-559/1163). Ces poèmes sont considérés comme un témoignage documentaire sur l'époque (les dernières années du califat fatimide) et sont confrontés à des écrits d'autres auteurs contemporains ou à d'autres écrits du poète lui-même, notamment ses " mémoires ", al-Nukat al-'așriyya fi aHbār alwuzară $\bar{a}^{\prime}$ al-mișriyya, édité au XIX` siècle par Hartwig Derenbourg ${ }^{1}$.

Rappelons très rapidement quelques données : poète, jurisconsulte sunnite et notable aisé, 'Umāra fuit le Yémen en 548/1154 suite à sa participation à des intrigues politiques. Il se réfugie à La Mecque, d'où il est envoyé à deux reprises, comme émissaire de l'émir al-Qāsim ibn Hišām, en Égypte, où il décide de s'installer suite à sa seconde mission. Intégré aux cercles du pouvoir fatimide, il sera le panégyriste de plusieurs vizirs, avant d'être exécuté par Saladin. Les causes réelles de son exécution, ses véritables convictions religieuses et doctrinales ou les premières années de sa vie au Yémen demeurent à ce jour entourées de nombreuses interrogations. Arrivé en Égypte 
l'année même où Ṭalā' i' ibn Ruzzīk (m. 556/1161) accède au vizirat, 'Umāra est surtout présenté dans l'ouvrage de Smoor comme le panégyriste des vizirs Ruzzīk ibn Țalā'i $i$ (m. 557/1162), puis Šāwar (vizir de de 1162 à 1163 puis de 1164 à 1169) et Ḍirgāam (m. 558/1164). Šāwar, qui avait renversé et exécuté Ruzzīk, fut à son tour renversé, quelques mois plus tard, par l'un de ses lieutenants, Ḍirḡām. Šāwar réintègrera sa fonction avec l'aide militaire de Nūr al-Dīn, auquel il s'était engagé à verser un tribut (ce qu'il ne fera pas), qui voyait dans l'appui qu'il lui apportait un moyen de contrer les visées d'Amaury I ${ }^{\circ}$. L'étude de Smoor commence lorsque Tală' i' organise le mariage qui causera sa perte, celui de sa fille avec l'enfant-Imam al-'Ádid (m. 567/1171), et s'arrête lorsque Šāwar accède, pour la seconde fois, au vizirat.

3 L'ouvrage, d'une facture technique impeccable ${ }^{2}$, se compose de six chapitres, dont les titres et les intertitres prennent surtout sens en référence aux thèmes des poèmes présentés et étudiés. Dans chaque chapitre (ou sous-chapitre), après une brève introduction de Smoor, rappelant quelques faits et présentant le poème ou les vers à venir, le texte arabe des vers de 'Umāra est reproduit. Quand le poème est intégral, il est précédé par l'énoncé : "The poem is now reproduced in its entirety (p. 91 et passim) ou une annonce analogue. Les citations poétiques varient en longueur et en diversité. Dans la plupart des cas et toujours pour les vers de 'Umāra, le texte arabe soumis à l'examen est d'abord donné dans son intégralité puis suivi par une traduction anglaise. Celle-ci est parfois entrecoupée ou suivie de commentaires (parfois paraphrastiques) de Pieter Smoor et assortie d'un solide apparat critique. Cependant, cet ordre de présentation (la citation intégrale en arabe puis sa traduction, avec ou sans commentaires) n'est pas toujours respecté. Certains poèmes sont fractionnés et le texte arabe est entrecoupé par la traduction (avec ou sans commentaires), ce découpage paraissant organisé autour du contenu thématique des vers. L'ouvrage propose, autour des mêmes thèmes, d'autres longues citations, poétiques ou prosaïques. Toutes les citations en prose sont données exclusivement dans leur traduction anglaise, contrairement à l'ensemble des citations poétiques, données en version bilingue.

4 La démarche suivie par l'auteur, les glissements ou les recoupements thématiques, rendent malaisé de rendre véritablement compte du contenu spécifique de chacun des chapitres. Il n'en est pas moins utile d'en donner une idée générale au lecteur, sous réserve que celui-ci garde à l'esprit que, dans la présentation qui suit, les « événements » mentionnés, quelle que soit leur réalité historique et factuelle, sont des données extraites de la poésie laudative ou des élégies funèbres composées par 'Umāra et qu'ils ne constituent que l'ossature principale de chaque chapitre, foisonnant par ailleurs d'autres informations.

5 Le chapitre 1, «The marriage of the vizier's daughter » (p. 12-71), traite en priorité de l'événement qui a conduit à l'assassinat de Țalā' $\mathrm{i}$ ' ibn Ruzzīk : l'union qu'il a contraint l'enfant-Imam al-'Āḍid, dernier calife fatimide, à contracter avec sa fille, un mariage somptueux par lequel il tentait d'avoir la mainmise sur le pouvoir fatimide.

6 Le chapitre 2, «Ruzzīk says farewell to Ṭalā’ $i$ " » (p. 73-161), traite en priorité de l'accès au pouvoir de Ruzzīk ibn Țală’'i $i^{i}$, rappelant qu'il avait déjà été désigné comme vicevizir, du vivant de son père, insistant sur l'importance et la puissance des Ruzzīk (Ruzzīk, son père Țalā' $i$ ', mais aussi son oncle, frère de son père, Badr) et sur les prises de position contradictoires des Fatimides à leur égard (ainsi, une tante d'al- 'Ā œuvré pour exécuter Țalā'i $i$ tandis qu'une autre œuvre à porter Ruzzīk au vizirat). Le courage militaire et les hauts faits des Ruzzỉk sont largement soulignés, notamment 
leur fermeté face aux actes de rébellion. Ce chapitre inclut également une sous-partie consacrée entièrement à l'étude d'un poème d'Ibn Qalāqis $(\mathrm{m} .1172)$ portant sur les mêmes thèmes.

7 Le chapitre 3 a pour titre « Ruzzīk et les parjures » (p. 163-201). Devenu vizir d'al- 'A did, Ruzzīk veut venger la mort de son père mais, selon les vers de 'Umāra, cette démarche ne doit en aucun cas constituer une mise en doute de la guidance assurée par l'imam, détenteur du Secret. Par contre, al-Mu'az̧zam ibn Qawām al-Dawla, l'instigateur du meurtre de Ṭală'i i', est semblable à Ibn Mulğam, l'assassin de 'Alī ibn Abī Ṭālib. Une partie de ce chapitre, plus volumineuse que celle qui lui est consacrée dans le précédent, porte sur Ibn Qalāqis et sa poésie. Le commentaire que celle-ci nécessite conduit $\mathrm{P}$. Smoor à la mettre en relation avec Mutanabbī en tant que poète et que prétendu prophète. La sanction des ennemis de Ṭală ${ }^{\prime} \mathrm{i}^{`}$ est aussi une occasion triste de se souvenir de ce dernier.

8 Dans le chapitre 4, "Ruzzìk enhances his position» (p. 203-267), sont présentés quelques poèmes qui traitent de Ruzzỉk jeune ( $\mathrm{y}$ compris du vivant de son père), exerçant ses responsabilités, que se soit dans sa fonction de substitut (niyāba), ou dans les mazālim. Ruzzīk (peut-être soucieux de s'en faire des alliés) aurait notamment fait libérer des émirs que son père avait préalablement fait emprisonner, il aurait aussi fait une importante donation à l'émir de La Mecque. La question du mariage de la sœur de Ruzzìk et de l'imam est de nouveau mentionnée et abordée dans une nouvelle série de vers. 'Umāra, quant à lui, insère dans les éloges de Ruzzīk, quelques mentions élégiaques sur son père disparu. Le chapitre accorde une large plage, à nouveau, au poète Ibn Qalāqis et introduit également, de manière substantielle, un autre contemporain des deux poètes, le célèbre secrétaire et conseiller de Saladin, al-Qāḍī alFādịil (m. 596/1199). Le sunnite 'Umāra, pressentant la fin des Fatimides, compose des poèmes pour demander la protection d'al-Qāḍi al-Fādil. Il compose également un panégyrique du savant et traditionniste sunnite, al-Ḥâfiz al-Silafī (installé à Alexandrie), auquel Ibn Qalāqis avait lui aussi dédié un poème. Bien que l'intitulé du chapitre puisse porter à penser à une puissance croissante de Ruzzìk, il est en réalité en perte de pouvoir, notamment en faveur de son général 'Izz al-Dīn Ḥusām, qui mate les rebelles. 'Umāra dédie à ce dernier quelques panégyriques (quoique les sources ne soient pas concordantes sur l'identité du dédicataire des poèmes en question). Enfin, ce chapitre met en exergue l'importance des augures dans la représentation qu'avaient du monde les personnages présentés.

Dans le chapitre 5, «Ruzzīk's screen and Shāwar's cave» (p. 269-306), la poésie laudative est aussi descriptive, et les tentures ou la tente y apparaissent comme un emblème du pouvoir. Sous l'influence de son entourage, Ruzzīk cherche à imposer son autorité à son général Šāwar, oubliant que son père lui avait conseillé, avant de mourir, de ne pas le provoquer. Le chapitre inclut également des vers dans lesquels on note un changement dans le discours de 'Umāra à propos de Ṭalā'i', dont il semble pouvoir désormais parler au passé comme une personne vraiment disparue. D’autres vers mentionnés ramènent le lecteur à l'époque ou Țală $i^{\prime}$, auquel ils sont destinés, vivait encore et faisait l'objet des panégyriques de 'Umāra. Le chapitre livre aussi le texte arabe d'un panégyrique d'al- 'Ậ̂did qui ne figurait pas dans l'édition Derenbourg. Enfin, on notera que le poète se plaint, de manière répétée, de ne pas être payé. Les vers confirment qu'il s'agit moins d'une récompense ponctuelle liée à la dédicace d'un poème que d'un salaire en tant que panégyriste en fonction. 

commence par un songe prémonitoire de Ruzzīk dans lequel il se trouve dans l'échoppe d'un marchand de têtes de mouton et voit un serpent entourer la lune. Selon l'interprétation ultime que lui en donne son cousin, la lune/vizir (donc Ruzzìk luimême) est encerclée par le serpent/armée, à cause des ambitions du marchand de têtes de mouton/Šāwar. Le chapitre traite dans une première phase de la confrontation qui aboutit à l'exécution de Ruzzīk et l'arrivée de Šāwar au vizirat, puis de la rébellion de Dirḡām contre le nouveau vizir qu'il renverse, enfin du retour au pouvoir de Šāwar. 'Umāra, en tant que panégyriste, suit le cours des événements et tente de leur adapter sa production poétique. Pour autant, il semble possible de noter qu'il montre beaucoup moins d'entrain à louer Ḍirḡām que les autres vizirs mentionnés. D'ailleurs, sitôt Šāwar revenu au pouvoir, le poète dépeindra Dirḡām comme un muhallil, cet époux provisoire de Wazāra, qui n'a d'autre fonction que de permettre à l'époux précédent de pouvoir s'unir de nouveau avec celle à qui il est destiné.

11 Ainsi, d'un chapitre à l'autre, "l'actualité » traitée est d'une densité très différente : alors que le premier chapitre tourne par exemple autour d'un seul événement (important certes), le mariage de l'Imam et de la fille du vizir, le dernier chapitre court sur trois vizirats successifs et les faits ayant conduit à leur avènement.

La démarche adoptée par Pieter Smoor s'inscrit en continuité avec ses précédents travaux, on pensera particulièrement à Kings and Bedouins in the Palace of Aleppo as Reflected in Ma'arri's Works, (Smoor, 1985, University of Manchester Press) : dégager, voire exhumer, dans une œuvre à caractère littéraire, des informations qu'il considère pertinentes sur la réalité factuelle, la vie quotidienne, les lieux et espaces, les personnages importants ou plus obscurs. Et les deux ouvrages sont marqués par la même minutie, la même érudition et la même précision. Dans ce sens, et par rapport à sa propre démarche, le travail de l'auteur est abouti. Dans cette perspective, il ne peut manquer d'enrichir les connaissances de tous ses lecteurs, de nombre de données et de détails, importants ou secondaire, indiscutables ou sujets à caution, en relation avec la fin de la dynastie fatimide et la poésie de cour de l'époque. Rappelons que les travaux de Pieter Smoor sur la poésie fatimide lui ont d'ailleurs valu le premier Prix Zahid Ali, une récompense quinquennale délivrée par l'Institute of Ismaili Studies.

Pour autant, quelques lecteurs, dont je suis, ne manqueront pas d'être déconcertés, voire réservés, sur plus d'un point. En effet, et quoique l'auteur signale, à plusieurs reprises, que la poésie n'est pas une source factuelle ou historique, c'est comme telle qu'elle paraît être traitée de fait, malgré quelques remarques incidentes à caractère rhétorique et stylistique. Cela pose d'autant plus question qu'il s'agit de poésie laudative, car les codes du panégyrique consistent in fine à "truquer " la réalité pour fabriquer la plus belle image possible de celui qu'on loue; c'est le domaine par excellence de l'exagération, de l'emphase et de l'hyperbole. Le discours moral d'une partie de la critique classique, qui préconisait un panégyrique qui ne s'écarte pas de la « vérité » est bien loin de la réalité des poèmes produits dans ce domaine. Pieter Smoor, dont on sait la vaste connaissance du patrimoine poétique ancien et de ses codes, ne l'ignore pas. Malgré cela, il choisit d'en extraire des données "réalistes » et «factuelles», et traite les «informations» fournies par ces poèmes comme des données sinon objectives, à tout le moins confinant à l'objectivité. Il est tout à fait dommage qu'il n'explique pas à son lecteur ce qui, selon lui, légitime cette démarche. 

pas à proprement parler de mise à distance critique ou de synthèse contradictoire qui permette d'évaluer véritablement les informations ainsi mises en lumière. En l'absence dans l'introduction (il n'y a pas de conclusion à l'ouvrage) d'informations sur l'approche défendue ou les lignes épistémologiques qui fondent le travail présenté, le lecteur a du mal à se situer et ne sait pas vraiment quel est le projet scientifique qui sous-tend la tâche si ample à laquelle l'auteur s'est assigné. Ces questions sont accentuées par le caractère parfois discontinu de l'exposé. Car rien n'est dit non plus sur la logique qui préside à la structure de chaque chapitre et plus généralement de l'ouvrage. Les retours fréquents sur des questions déjà traitées, les fluctuations du continuum chronologique et les ouvertures ponctuelles vers d'autres œuvres ou périodes littéraires brouillent les cartes. A titre d'exemple (pour n'en citer qu'un seul), pourquoi la traduction du poème de 'Umāra a-t-elle été interrompue p. 212 par une référence comparative à Ibn al-Rūmī (quoique je souscrive pleinement à la grande beauté de la citation retenue) ? Certes, l'intertextualité est parfaitement légitime, mais qu'est-ce qui justifie qu'elle soit mise en œuvre ici et pas ailleurs, ou à chaque fois qu'elle le pourrait? Cet excursus ou d'autres de même type sont d'autant plus inattendus que l'ouvrage ne traite pas d'histoire littéraire ni de diachronie.

Sans vouloir engager ici un débat philosophique sur le «tout fiction » versus «tout réalité » et sans nier la richesse de cet ouvrage et l'érudition qui le porte, il n'en demeure pas moins que Wazāra, the killer of many husbands aurait certainement gagné à mettre explicitement les textes qui y sont étudiés à l'épreuve des théories de notre temps, qu'elles soient littéraires ou historiques, dans le but de dissocier le regard du chercheur de celui du poète. L'importance même des informations mises en évidence, pour la connaissance des détails de l'histoire de la fin de la dynastie fatimide, le nécessitait et le justifiait.

\section{NOTES}

1. L'édition Derenbourg est consultable en ligne sur www.alwarraq.net ou téléchargeable en format PDF sur http://www.al-mostafa.info/data/arabic/depot3/gap.php?file=i003602.pdf. Sauf pour un poème, publié par Pieter Smoor dans le chapitre 5, Derenbourg est également l'éditeur de l'œuvre poétique de 'Umāra dans Poésies, Épitres, Biographies, Notices en Arabe par 'Oumâra et sur 'Oumâra (Paris : Leroux, 1902, Publications de l'École des Langues Orientales Vivantes).

2. J'ai relevé une unique coquille, p. 124 (metes pour meets) et je tiens à souligner l'agrément qu'éprouve le lecteur devant la mise en page des poèmes en arabe, qui pourrait servir de modèle à nombre d'éditeurs anglo-saxons qui, quand ils ne refusent pas purement et simplement de publier des citations qui ne soient pas en caractères latins, malmènent la présentation des vers classiques, notamment par une présentation si irrégulière de la longueur des hémistiches qu'ils en deviennent illisibles.

Bulletin d'études orientales, Tome LIX | octobre 2010 


\section{AUTEURS}

\section{KATIA ZAKHARIA}

Université de Lyon 2 et GREMMO UMR 5195 